\section{Mit Inhaler geht am meisten schief}

Ob Patienten ihre Medikamente richtig einnehmen, hängt von der Dosierhilfe ab. In einer Studie mit 233 Heimbewohnern verdoppelte sich die Fehlerquote, wenn Tabletten ohne Dispenser ausgegeben wurden. Bei anderen Formen lag die Quote noch höher. Alldred DP et al, BMJ Qual Saf 2011, 20:397

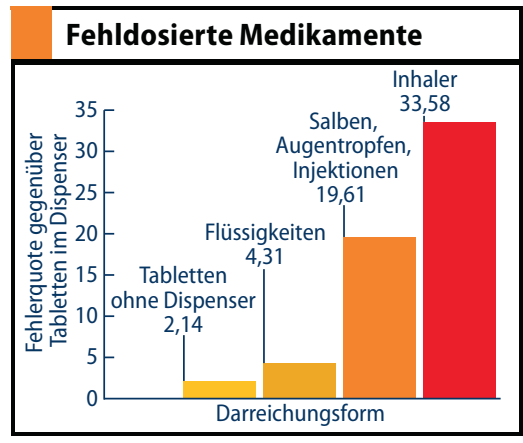

\section{Erdnuss-Schock aus der Blutkonserve}

Nicht nur im Essen, auch in Blutprodukten können Nahrungsmittelallergene auftauchen. Dies zeigte der Fall eines sechsjährigen Jungen, bei dem eine Thrombozytentransfusion eine anaphylaktische Reaktion auslöste. Die Ärzte fanden heraus, dass drei der Spender am Abend vor der Blutentnahme Erdnüsse gegessen hatten, was der Junge seit seinem zweiten Lebensjahr streng vermied. Im Blut des Kindes konnten dann tatsächlich Antikörper gegen das Hauptallergen der Erdnüsse nachgewiesen werden. Jacobs JF et al, NEJM 2011, 364:1981

\section{springermedizin.de via Twitter folgen}

Der Sommer ist da und das Zwitschern geht immer weiter: Werden Sie zum "Follower" und "lauschen" Sie uns bei Twitter. twitter.com/springermedizin
Falsche Vorsicht schadet Keine Angst vorm Betablocker bei COPD!
Wenn Patienten mit einer kardiovaskulären Erkrankung gleichzeitig an einer COPD leiden, vermeiden viele Ärzte die Verordnung eines Betablockers. Sie befürchten, dass Betablocker die Wirkung der inhalativen Betamimetika konterkarieren und einen Bronchospasmus auslösen könnten. Diese Vorsicht ist nicht nur unbegründet, sie schadet den Patienten sogar.

In einer retrospektiven Studie aus Schottland war eine Betablockertherapie mit einer niedrigeren Sterblichkeit und überraschenderweise auch mit einem geringeren Risiko für COPD-Exazerbationen assoziiert. Für die Studie wurden Registerdaten von 5977 COPD-Patienten, die meisten in den GOLD-Stadien II oder III, ausgewertet. Alle Patienten wurden mit inhalativen Bronchodilatatoren behandelt, ein Teil auch mit inha- lativen Kortikosteroiden. 819 Patienten erhielten wegen einer kardiovaskulären Erkrankung einen Betablocker, davon 720 einen kardioselektiven Wirkstoff.

\section{Weniger Notfälle und Krankenhauseinweisungen}

Nach einer Beobachtungsdauer von 4,3 Jahren lag die Sterblichkeit bei den Patienten unter zusätzlicher Betablockade um 22\% niedriger. Der Überlebensvorteil zeigte sich unabhängig von der begleitenden COPD-Therapie. Betablocker erwiesen sich auch in Bezug auf COPD-Exazerbationen als vorteilhaft: Sowohl notfallmäßige Verordnungen von oralen Kortikosteroiden als auch respiratorisch bedingte Krankenhauseinweisungen waren seltener als in den Vergleichsgruppen ohne Betablocker.

Short PM et al, BMJ 2011, 342:d2549

\section{Leider nur bei Männern ... Ehe lindert Herzinfarkt}

\section{Die raschere stationäre Versorgung könnte ein Grund für die Beobachtung sein, dass das Mortalitätsrisiko ver- heirateter Männer mit akutem Herzin- farkt niedriger ist als das von allein le- benden männlichen Infarktpatienten.}

Die Arbeitsgruppe um Dr. Clare Atzema aus Toronto hat in einer Studie bei 4403 Personen mit einem von Brustschmerz begleitetem Myokardinfarkt retrospektiv untersucht, wie viel Zeit jeweils zwischen Symptombeginn und der Aufnahme in eine Klinik vergangen war. Differenziert wurde danach, ob diese
Personen in einer Ehe oder eheähnlichen Gemeinschaft oder als Single lebten.

\section{Verspätet in die Notaufnahme}

Das Risiko, erst mit einer mehr als sechsstündigen Verspätung in die Notaufnahme zu gelangen, war allerdings nur bei verheirateten Männern im Vergleich $\mathrm{zu}$ allein lebenden Männern statistisch signifikant niedriger. Bei Frauen war der Ehestatus dagegen in dieser Studie nicht mit einer signifikant rascheren Einlieferung in eine Klinik assoziiert. 